\title{
Editorial
}

Patrick J. McGrath PH D

Gary G. Johnson MD FRCPC

\section{Pain management in children}

Pain in paediatric patients has, after a long period of neglect, begun to receive the research and clinical attention it deserves. ${ }^{1-6}$ As well, changes have occurred in the knowledge, sophistication and expectations of the public. $^{7-8}$

There are four aspects of paediatric pain that are particularly relevant to anaesthesia, because of recent developements and because of the probability that important advances will be made in the next few years. The four areas are: mapping and understanding of the development of pain pathways; pain measurement; management of pain associated with surgery; and controlling pain from aversive medical procedures.

Work on animals and humans has begun to clarify the development of pain mechanisms in the neonate. Mapping of the anatomical, neurochemical, physiological and behavioural correlates of pain has been rapid in the past few years. ${ }^{9}$

Some recent work ${ }^{10,11}$ suggests that pain pathways in the neonate (rat and human) may not be functionally developed until some time following birth and that premature neonates may be particularly sensitive to aversive procedures because of the immaturity of descending inhibitory pain control mechanisms.

Progress has recently been made in the development and validation of pain measures. Obviously, this is important because measurement is the prerequisite of scientific advances. Reliable and valid measures of pain by self report, ${ }^{12-14}$ by behavioural observations, ${ }^{15-17}$ and by physiological and biochemical measures ${ }^{18-19}$ have been developed. As a result, the clinician and researcher huve a wide array of well-validared tools with which to measure pain in children. In fact, now there are more wellvalidated measures of pain in children than there are in

From the Departments of Psychology and Paediatrics (PJM), University of Ottawa, and the Department of Anacsthesia, Children's Hospital of Eastern Ontario, and University of Ottawa (GGJ) Ottawa, Ontario.

Address correspondence to: Dr. G.G. Johnson, Department of Anacsthesia, Children's Hospital of Eastern Ontario, 401 Smyth Road, Ottawa, Ontario, K1H 8L1. adults. This is as it should be, because the constantly developing cognitive, behavioural and physiological capacities of children as they mature demand that a variety of measures be available. There are, however, serious gaps in our knowledge of pain measurement. In particular, there is room for considerable development of pain measures for the neonate, measures for the physically and mentally handicapped and measures of aspects of pain other than pain severity in children.

Both pain or distress during surgery and pain following surgery have received attention. Recent studies have demonstrated that there are effective methods to control pain during surgery, even in unstable or critically ill neonates. ${ }^{20.21}$ Adequate analgesia during surgery reduces the stress response and may result in better clinical outcome ${ }^{20-22}$ As well, there has been a beginning of much needed and well designed research on postoperative pain. ${ }_{-}^{23-25}$ The report by Maunuksela ef al in this issue, ${ }^{25}$ which evaluates the use of prophylactic intravenous indomethacin, is a good example of this new area of investigation. The use of intravenous infusions on the wards may not be the most practical or effective route. Moreover, the non-steroidal anti-inflammatories appear not to be potent enough for immediate postoperative pain. In the study of Maunuksela et al. the vast majority of the children, even in the experimental group, were not pain-free.

Recently, there has been more widespread use of regional anaesthesia and nerve blocks, both alone and as a supplement to general anaesthesia in children and neonates ${ }^{26}$ There is also interest in the use of long-lasting opioids administered perioperatively for postoperative pain relief. As Maunuksela et al. note, a great deal more needs to be done. Properly designed trials must be undertaken on the whole range of analgesics, investigating a variety of schedules, routes of administration and methods of titration of doses.

Some children, such as sick neonates and children with cancer, experience a great many aversive procedures such as venipuncture, lumbar puncture, arterial blood sampling and bone marrow aspiration, usually without any significant pain control. Although much work has been published on teaching children hypnotic techniques 
to reduce distress, ${ }^{27}$ relief is by no means complete, the practice is not widespread and, in many cases, is impractical. Medical interventions to reduce pain during aversive procedures appear to have been more common in Europe but few evaluations have been published. The Finnish group headed by Maunuksela described the use of flunitrazepam in conjunction with fentanyl for bone marrow aspiration and needle biopsy in children over the age of 3 years. ${ }^{28}$ Similarly they have reported on the use of lidocaine/prilocaine cream for venous cannulation in children. ${ }^{29}$ Other reports have described analgesia for hydrostatic reduction of ileocolic intussusception. ${ }^{30}$

Anaesthetists have much to offer in both the research and management of pain in children. There is a need for more studies such as those of Maunuksela et al. to develop and evaluate better methods to manage paediatric pain.

Clinical practice must implement what is already known about pain in children, since adequate analgesia is now not only a humanitarian imperative, but also the expected standard of medical practice. Children deserve no less.

\section{Le contrôle de la douleur chez l'enfant}

Aprês avoir été négligée longtemps, la douleur chez le groupe de patients pédiatriques commence à recevoir l'attention qu'elle mérite de la par des chercheurs et cliniciens. ${ }^{1-6}$ On assiste ainsi à des progrès dans nos connaissances, et dans la sophistication et les expectatives du public. ${ }^{7-8}$

Quatre aspects de la douleur pediatrique particulièrement pertinents à l'anesthésie ressortent avec les récents développements et la possibilité de progrès importants dans les prochaines années. Ces quatre secteurs sont: le "mapping" et la compréhension des différentes voies de la douleur; la quantification de la douleur; le contrôle de la douleur associée à la chirurgie; et le contrôle de la douleur lors des procédures médicales invasives.

Des études tant chez les humains que chez les animaux ont commencé à clarifier les mécanismes de la douleur chez le nouveau-né. Les études anatomiques, neurochimiques, physiologiques, et du comportement concernant la douleur se sont multipliées rapidement dans les demières annees. ${ }^{9}$

Des études récentes ${ }^{t 0,11}$ suggèrent que les voies de la douleur chez le nouveau-né (rat et hurnain) peuvent ne pas être fonctionnellement développées jusqu'aptès la naissance. Des nouveaux-nés prématurés peuvent ainsi être particulièrement sensibles à des procédures invasives à cause de l'immaturité des voies déscendantes de contrôle de la douleur.

Du progrès a été récemment acquis dans le développement et la validation de la quantification de la douleur. Il est évident que ceci est jimportant car la quantification est un pré-requis pour le progrès scientifique. Des mesures valides et fiables de la douleur par déclaration subjective, ${ }^{12.14}$ par des observations du comportement ${ }^{15,17} \mathrm{et}$ par des mesures physiologiques et biochimiques ont été développées. ${ }^{18,19}$ Ainsi le clinicien et le chercheur ont un assortiment varié d'outils valides avec lesquels ils peuvent mesurer la douleur chez les enfants. En fait, il existe maintenant plus de moyens valides pour l'enfant qu'il en existe pour ladulte. Ceci doit en être ainsi, car les développements constants dans les capacités cognitives, physiologiques et des comportements chez l'enfant eu maturation exigent qu'une variété de mesures soit disponible. Il existe cependant encore des lacunes sérieuses dans nos moyens de mesure de la douleur. En particulier, il y a ainsi encore de la place pour un développement considérable dans la quantification de la douleur chez le nouveau-né, l'handicapé mental et physique. La mesure des différents aspects de la douleur chez l'enfant autre que sa sévérité doit aussi être plus étudiée.

Tant la douleur que la détresse lors de la chirurgie ainsi que la douleur post chirurgie ont attiré beaucoup d'attention. Des études récentes ont démontré que l'on possède des moyens efficaces pour contrôler la douleur lors de la chinurgie, même chez les nouveaux-nés instables ou critiquement malades. ${ }^{20,21}$ Une analgésie adéquate lors de la chirurgie réduit la réponse au stress et peut provoquer une issue clinique plus favorable. ${ }^{20-22}$ On a commencé aussi des études tant attendues et bien conçues sur la douleur post-opératoire. ${ }^{23-25}$ L'étude de Maunuksela et al. dans ce numéro ${ }^{25}$ évaluant l'usage prophylactique de l'indométhacine intraveineuse est un bon exemple pour ce secteur d'investigation. L'utilisation de perfusion sur l'êtage peut ne pas être le plus pratique ou le plus efficace des moyens. De plus les anti-inflammatoires non stérödaux ne semblent pas aussi puissants pour contrôler la douleur post-opératoire immédiate. Dans cette étude de Maunuksela et al., la vaste majorité des enfants, même du groupe expérimental, n'était pas soulagée de leur douleur.

Récemment, il y a eu un usage étendu de l'anesthésie régionale ct des bloes nerveux, scule ou en supplément à $l^{\prime}$ anesthésie générale chez les enfants et nouveaux-nés. ${ }^{26}$ Il y a aussi un intérêt dans l'utilisation des opiacés à longue duré d'action administrés en période périopéra- 
toire pour le contrôle de la douleur postopératoire. Comme le dit Maunuksela et al. il reste bcaucoup de choses à faire dans ce domaine. Des études bien conçues doivent être faites pour englober tous les analgésiques, investiguer une variété de cédules, voies d'administration ct moyens d'ajuster les doses.

Quelques enfants, comme les nouveaux-nés malades et les enfants atteints de cancer, subissent plusieurs procédures invasives telles que ponction veineuse, ponction lombaire, ponction artérielle et aspiration de la moëlle habituellement sans contrôle significatif de la douleur. Même si plusieurs travaux ont été publiés sur 1'apprentissage des enfants de techniques hypnotiques afin de réduire la détresse ${ }^{27}$ le soulagement n'est certainement pas complet. D'autre part l'utilisation de cette technique n'est pas étendue et dans plusieurs cas elle n'est pas pratique. Les interventions médicales afin de réduire la douleur durant des procédures invasives sont apparemment plus fréquentes en Europe mais peu d'études pouvant les évaluer ont été publiées. Le groupe de Finlande dirigé par Maunuksela a décrit l'utilisation du flunitrazépam en conjonction avec le fentanyl pour la biopsie médullaire chez les enfants âgés de plus de trois ans. ${ }^{2 \mathrm{~B}}$ Ils ont aussi rapporte l'utilisation de la crème de lidocaïne-prilocaïne pour les canulations veineuses chez l'enfant. ${ }^{29}$ D'autres études ont décrit des moyens d'analgésie pour la réduction hydrostatique de l'invagination iléocolique. ${ }^{30}$

Les anesthésistes ont beaucoup à offrir tant dans la recherche que dans la conduite clinique du contrôle de la douleur chez les enfants. Plusieurs études comme celles de Maunuksela $e t$ al. sont nécessaires afin de développer et d'évaluer des meilleurs moyens pour contrôler la douleur chez le groupe pédiatrique.

En clinique on doit mettre en pratique ce qui est déjà connu sur la douleur chez les enfants car une analgésie adéquate est actuellement non seulement un impératif humanitaire mais aussi une norme de notre pratique médicale. Nos enfants n'en méritent pas moins.

\section{References}

1 Berry FA, Gregory GA. Do premature infants require anesthesia for surgery? Anesthesiology 1987; 67: 291-3.

2 Lancet, Pain, anaesthesia and babies. 1987; 11: 543-44.

3 McGrath PJ, Unruh AM. Pain in children and adolescents. Amsterdam: Elsevier, 1987

4 Polans RL, Roberts RI, Gutierrez-Moxorra JF, Fonkalsrud, $E W$. Neonatal anesthesia. Pediatrics 1987; 80: 446.

5 Schechter NL. Pain and pain control in children. 1985; Curnent Problems in Pediatrics.

6 Yaster $M$. Analgesia and anesthesia in neonates. J Pedjatr 1987; 111: 394-6.
7 Fischer A Babies in pain. Redbook, 1987; October $124-5,184,186$.

8 Boffey $P M$. Infants' sense of pain is recognized, finally. New Yort Times, 1987; November 24: C3.

9 Arand KJS, Hickey PR. Pain and its effects in the human fetus and neonate, N Engl J Med 1987; 317: 1321-9.

10 Fitzerald $M$, Kotzenburg $M$. The functional development of descending inhibitory pathways in the dorsolateral funiculus of the newborn rat spinal cord. Development Brain Research 1986; 24: 261-70.

1 Fizgerald $M$, Shaw A, MacIntosh $N$. The postnatal deve]opment of the cutaneous flexor reflex: a comparutive study in premature infants and newborn rat pups. Dev Med Child Neurol (in press).

12 LeBaron S, Zeitzer $L$. Assessment of acute pain and anxiety in children and adolescents by self reports, observer reports, and a behavior checklist. J Consult Clin Psychol 1984; 52: 729-38.

13 Beyer JE, Aradine CR. Content yalidity of an instrument to measure young children's perception of their pain. $J$ Pediatr Nurs 1986; 1 : 386-95

14 Maumuksela E-L, Olkkola KT, Korpela R. Mcasurement of pain in children with self-reporting and behavioral assessment. Clin Pharm Therap 1987; 42: 137-41.

15 McGrath PJ, Johnson G, Goodman JT, Schillinger J, Dunn J, Chapman J. The CHEOPS: a behavioral scale to measure post operative pain in children. In: Fields HL, Dubner R, Cervero F (Eds). Advances in Pain Research and Therapy. New York: Raven Press, 1985. 395-402.

16 Grumau RVE, Craig $K$. Pain expression in neonates: facia] action and cry. Pain 1987; 28: 395-410.

17 Katz ER, Kellerman J, Siegel SE. Distress behavior in children with cancer undergoing medical procedures: developmental considerations. J Consult Clin Psychol 1980; 48: 356-65.

18 Anand KJS, Aynsley-Green A. Metabolic and endocrine effects of surgical ligation of patent ductus arteriosus in the human preterm neonate: are there implications for further improvement of postoperative outcome? Mod Probl Paediatr 1985; 23, 143-57.

19 Williamson PS, Williamson ML. Physiologic stress reduction by a local anesthetic during newborn circumcision. Pediatrics 1983; 71: 36-40.

20 Anand KJS, Sippell WG, Aynsley-Green A. Randomized trial of fentanyl anaesthesia in preterm neonates undergoing surgery: effects on the stresss response. Lancet 1987; 1: 243-7.

21 Anand KIS, Sippell WG, Aynsley-Green A. Does the newborn infant require anaesthesia during surgery? Answers from a randomized trial of halothane anaesthesia. 1987; Vth World Congress on Pain, Hamburg. 
22 Waugh $R$, Johnson GG. Current considerations in neonatal anaesthesia. Can Anaesth Soc J 1984; 31:700-9.

23 O'Hara M, McGrath PJ, D'Astous $J$, Vair C. Oral morphine versus injected meperidine (demerol) for pain relief after orthopedic surgery. J Pediatr Orthoped 1987; 7; $78-82$.

24 Langer $J$, Shandling $B$, Rosenberg $M$. Intraoperative bupivicaine during outpatient hemia repair in children: a randomized double blind trial. J Ped Surg 1987; 22, 267-70.

25 Maunuksela E-L, Olkkola KT, Korpela R. Does prophylactic intravenous infusion of indomethacin improve the management of postoperative pain in children? Can $J$ Anaesth 1988; 35: 123-7.

26 Broadman $L M$. Pediatric regional anesthesia and postoperative analgesia. in: Barash PG (Ed). American Society of Anesthesiologists. Refresher Courses in Anesthesiology Volume 14, Philadelphia: J.B. Lippincott, pp 43-60, 1986.

27 Zeltzer L, LeBaron SA Hypnosis and nonhypnutic techniques for reduction of pain and anxiety during painful procedures in children and adolescents with cancer. $J$ Pediatr 1982; 101, 1032-5.

28 Maunuksela E-L, Rajantie J, Siimes MA. Flunitrazepamfentanyl induced sedation and analgesia for bone marrow aspiration and needle biopsy in children. Acta Anaesthesiol Scand 1986; 30, 409-11.

29 Maunuksela E-L, Korpela R. Double blind evaluation of a lignocaine-prilocaine cream (EMLA) in children: effect on the pain associaled with venous cannulation. Br J Anaesth 1986; 58: 1242-5

30 Touloukian RJ, O' Connell JB. Markowitz RI. Rosenfield $N$, Seashore JH, Ablow RC. Analgesic premedication in the management of ileocolic intussusception. Pediatrics 1987; 79: 432-4. 